Supporting Information: "Performing Molecular Dynamics Simulations and Computing Hydration Free Energies on the B3LYP-D3(BJ) Potential Energy Surface with Adaptive Force Matching: a Benchmark Study with Seven Alcohols and One Amine"

\author{
Dong Zheng, ${ }^{1}$ and Feng Wang, ${ }^{1, *}$ \\ ${ }^{1}$ Department of Chemistry and Biochemistry, University of Arkansas, Fayetteville, Arkansas, \\ 72701, USA
}




\section{The $\mathrm{QM} / \mathrm{MM}$ region selection protocol}

With this protocol, the QM region is composed of a center region and a boundary region, although both the center and boundary regions are modeled quantum mechanically, only the atoms in the center region are used for force matching.

For each conformation selected from the sampling step, the QM/MM configuration is constructed with following procedure:

1. The solute will be in the QM center region. Any water molecule with at least one atom within $3 \AA$ from the solute will be defined as a first hydration shell water molecule and included in the QM region.

2. Five water molecules are chosen randomly from the first hydration shell water molecules identified in step one and included in the center region. Any water within $2.6 \AA$ of these five water molecules will also be included in the QM region.

3. Any water with at least one atom within $9 \AA$ of the solute but not in the QM region will be kept as MM particles. Water molecules further away will be discarded.

4. Water molecules in the QM region but not in the center region will be in the boundary region. 


\section{Force filed parameters}

Table S1. All force field parameters for ethanol.

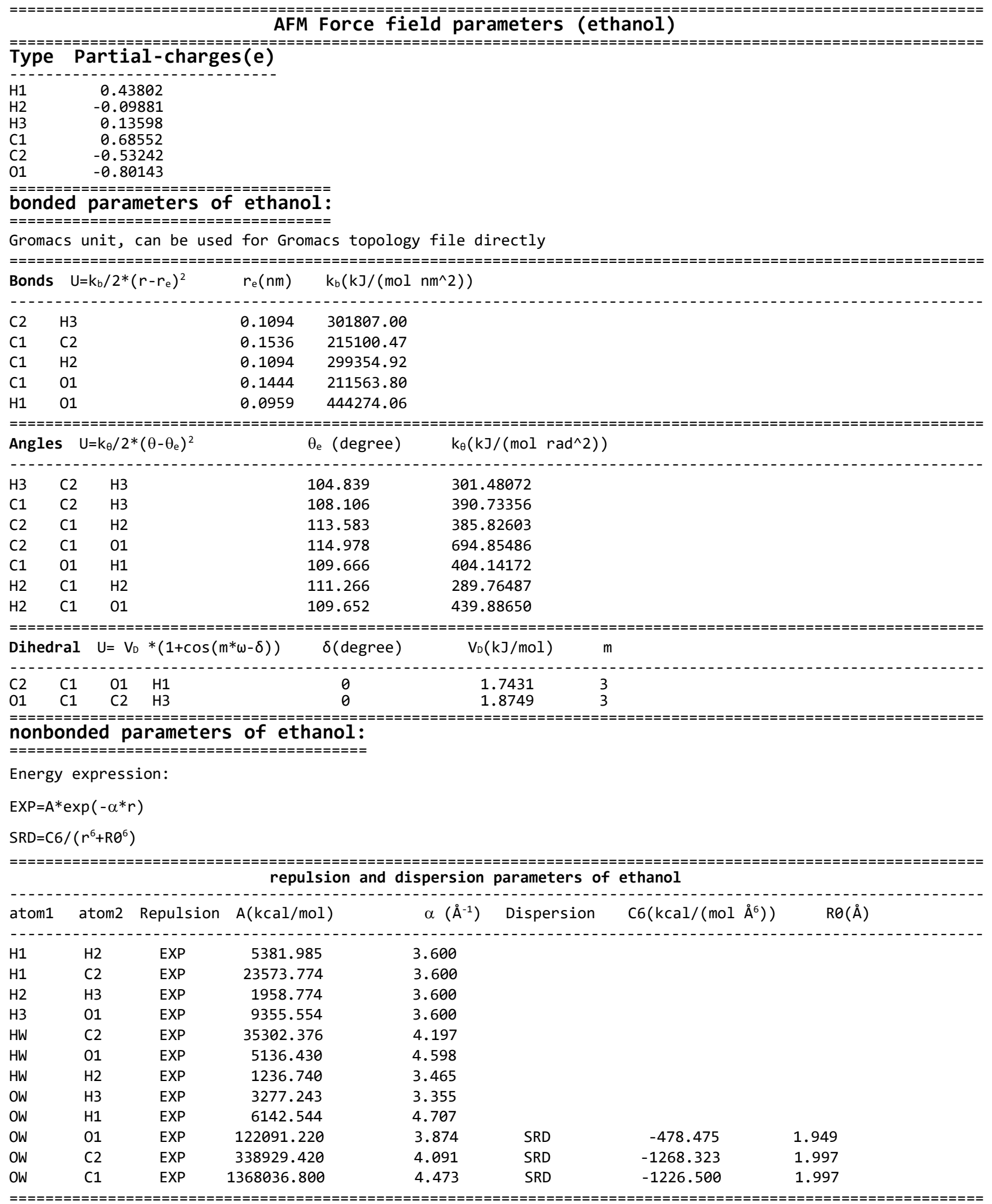


Table S2. All force field parameters for isobutanol

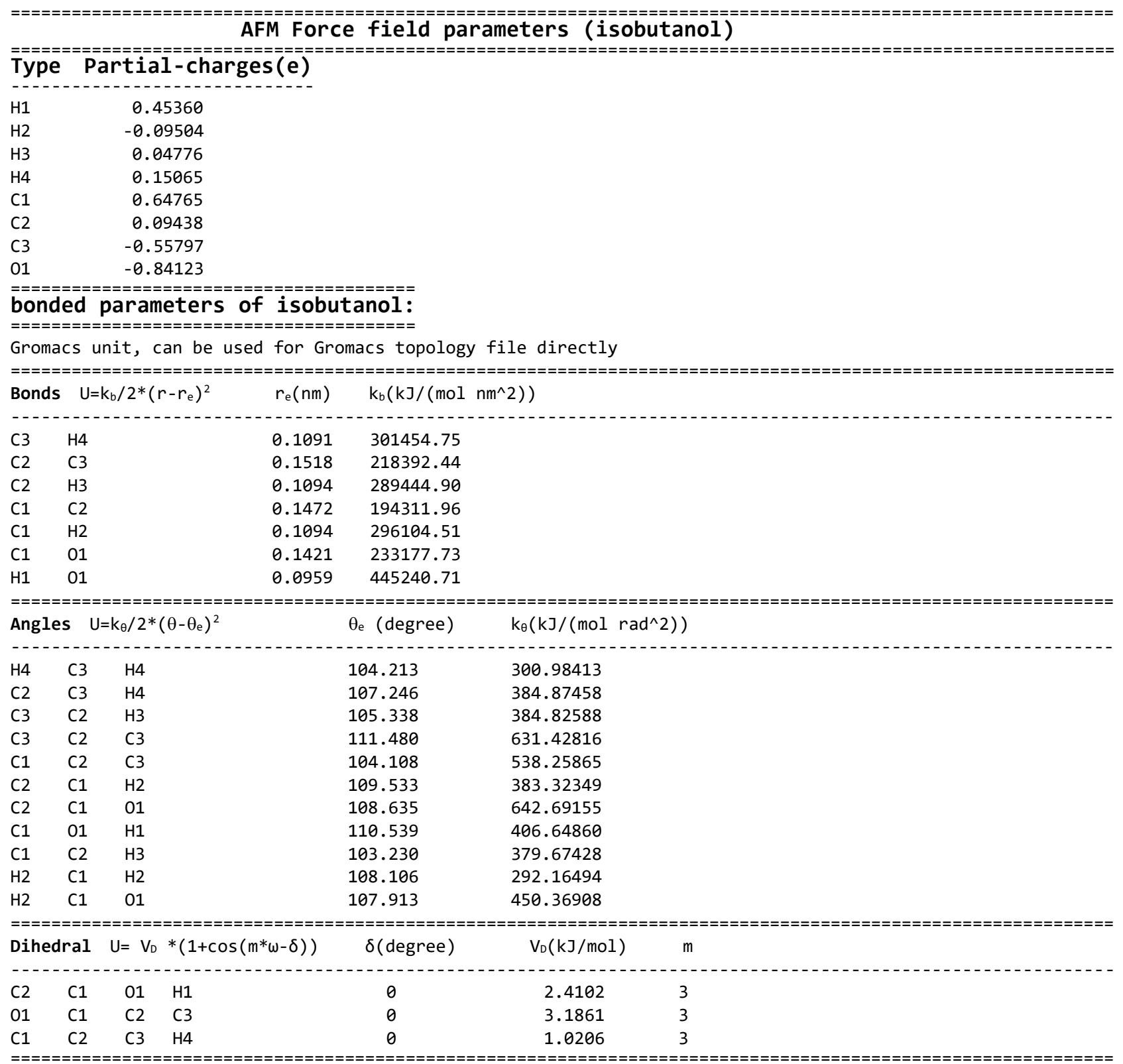

$========================================+$
nonbonded parameters of isobutanol:

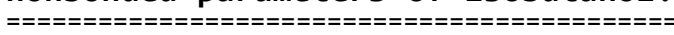

Energy expression:

$\operatorname{EXP}=A^{*} \exp \left(-\alpha^{*} r\right)$

$\mathrm{SRD}=\mathrm{C} 6 /\left(\mathrm{r}^{6}+\mathrm{R}^{6}\right)$

repulsion and dispersion parameters of isobutanol

\begin{tabular}{|c|c|c|c|c|c|c|c|}
\hline atom1 & atom2 & Repulsion & $\mathrm{A}(\mathrm{kcal} / \mathrm{mol})$ & $\alpha\left(\AA^{-1}\right)$ & Dispersion & $\mathrm{c} 6\left(\mathrm{kcal} /\left(\operatorname{mol} \AA^{6}\right)\right)$ & $\mathrm{R} \theta(\AA)$ \\
\hline $\mathrm{H} 1$ & $\mathrm{H} 2$ & EXP & 4964.579 & 3.600 & & & \\
\hline H1 & $\mathrm{C} 3$ & EXP & 20497.154 & 3.600 & & & \\
\hline $\mathrm{H} 2$ & H3 & EXP & 1475.683 & 3.600 & & & \\
\hline $\mathrm{H} 2$ & $\mathrm{H} 4$ & EXP & 448.687 & 3.600 & & & \\
\hline H3 & 01 & EXP & 6803.311 & 3.600 & & & \\
\hline $\mathrm{H} 4$ & 01 & EXP & 5888.671 & 3.600 & & & \\
\hline C3 & 01 & & & & SRD & -699.913 & 1.997 \\
\hline HW & $\mathrm{C} 3$ & EXP & 7849.855 & 3.667 & & & \\
\hline HW & 01 & EXP & 1551.003 & 3.697 & & & \\
\hline $\mathrm{HW}$ & $\mathrm{H} 2$ & EXP & 881.973 & 3.367 & & & \\
\hline OW & $\mathrm{H} 4$ & EXP & 6036.912 & 3.629 & & & \\
\hline OW & H3 & EXP & 8796.591 & 3.708 & & & \\
\hline OW & $\mathrm{H} 1$ & EXP & 679.660 & 3.241 & & & \\
\hline
\end{tabular}




$\begin{array}{rrrrrrr}\text { OW } & \text { O1 } & \text { EXP } & 303390.800 & 4.268 & \text { SRD } & -465.858 \\ \text { OW } & \text { C3 } & \text { EXP } & 120451.440 & 3.728 & \text { SRD } & -1263.338 \\ \text { OW } & \text { C2 } & \text { EXP } & 3109287.600 & 4.916 & \text { SRD } & -828.355 \\ \text { OW } & \text { C1 } & \text { EXP } & 155536.290 & 3.686 & \text { SRD } & 1.997 \\ & & & -1207.374 & 1.997\end{array}$


Table S3. All force field parameters for 2-butanol

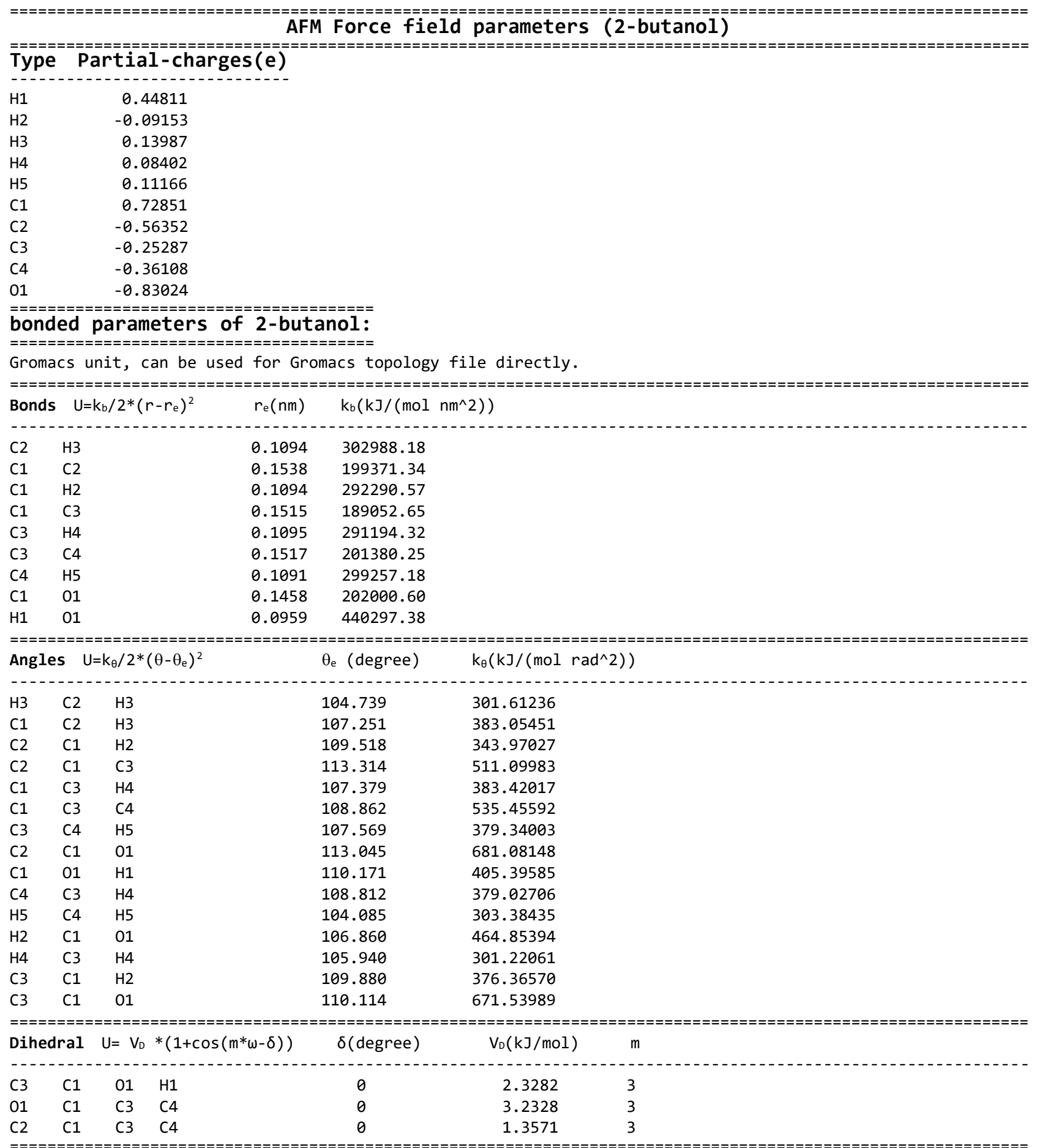

$======================================$
nonbonded parameters of 2-butanol:

$===================================0$

Energy expression:

$\operatorname{EXP}=A^{*} \exp \left(-\alpha^{*} r\right)$

$\mathrm{SRD}=\mathrm{C} 6 /\left(\mathrm{r}^{6}+\mathrm{R}^{6}\right)$

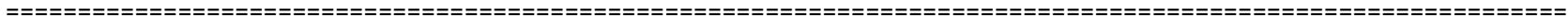
repulsion and dispersion parameters of 2-butanol

\begin{tabular}{|c|c|c|c|c|c|c|}
\hline atom1 & atom2 & Repulsion & $\mathrm{A}(\mathrm{kcal} / \mathrm{mol})$ & $\alpha\left(\AA^{-1}\right)$ & Dispersion & $\mathrm{c} 6\left(\mathrm{kcal} /\left(\operatorname{mol} \AA^{6}\right)\right)$ \\
\hline 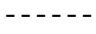 & --- & - & 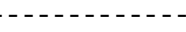 & ------- & & \\
\hline $\mathrm{H} 1$ & $\mathrm{H} 2$ & EXP & 4995.222 & 3.600 & & \\
\hline $\mathrm{H} 1$ & $\mathrm{C} 2$ & EXP & 20865.609 & 3.600 & & \\
\hline $\mathrm{H} 1$ & C3 & EXP & 17437.310 & 3.600 & & \\
\hline $\mathrm{H} 1$ & $\mathrm{C} 4$ & EXP & 15022.134 & 3.600 & & \\
\hline $\mathrm{H} 2$ & H3 & EXP & 3716.633 & 3.600 & & \\
\hline
\end{tabular}




\begin{tabular}{|c|c|c|c|c|c|c|c|}
\hline $\mathrm{H} 2$ & $\mathrm{H} 4$ & EXP & 1336.325 & 3.600 & & & \\
\hline $\mathrm{H} 2$ & H5 & EXP & 510.966 & 3.600 & & & \\
\hline H3 & 01 & EXP & 9125.924 & 3.600 & & & \\
\hline H3 & C3 & EXP & 13066.093 & 3.600 & & & \\
\hline H3 & C4 & EXP & 9583.179 & 3.600 & & & \\
\hline $\mathrm{H} 4$ & 01 & EXP & 5838.290 & 3.600 & & & \\
\hline $\mathrm{H} 4$ & C2 & EXP & 10122.768 & 3.600 & & & \\
\hline $\mathrm{H} 5$ & 01 & EXP & 2501.525 & 3.600 & & & \\
\hline $\mathrm{H} 5$ & C2 & EXP & 7049.564 & 3.600 & & & \\
\hline 01 & C4 & EXP & 25670.937 & 3.600 & SRD & -848.406 & 1.997 \\
\hline C2 & C4 & EXP & 56393.233 & 3.600 & SRD & -2043.149 & 2.045 \\
\hline $\mathrm{HW}$ & C4 & EXP & 2142.718 & 3.205 & & & \\
\hline $\mathrm{HW}$ & C3 & EXP & 3882.156 & 3.366 & & & \\
\hline $\mathrm{HW}$ & C2 & EXP & 15461.000 & 3.780 & & & \\
\hline $\mathrm{HW}$ & 01 & EXP & 3550.930 & 4.414 & & & \\
\hline OW & H5 & EXP & 4690.037 & 3.514 & & & \\
\hline OW & $\mathrm{H} 4$ & EXP & 4317.384 & 3.457 & & & \\
\hline OW & $\mathrm{H} 3$ & EXP & 4197.151 & 3.476 & & & \\
\hline $\mathrm{HW}$ & $\mathrm{H} 2$ & EXP & 760.426 & 3.247 & & & \\
\hline OW & $\mathrm{H} 1$ & EXP & 1938.433 & 4.080 & & & \\
\hline OW & 01 & EXP & 138107.770 & 3.891 & SRD & -464.361 & 1.949 \\
\hline OW & C4 & EXP & 202794.540 & 3.910 & SRD & -1345.432 & 1.997 \\
\hline OW & C3 & EXP & 2442305.400 & 4.821 & SRD & -1008.673 & 1.997 \\
\hline OW & C2 & EXP & 117628.030 & 3.751 & SRD & -1265.442 & 1.997 \\
\hline ow & C1 & EXP & 858050.560 & 4.292 & SRD & -1135.526 & 1.997 \\
\hline
\end{tabular}


Table S4. All force field parameters for 1,2-butanediol

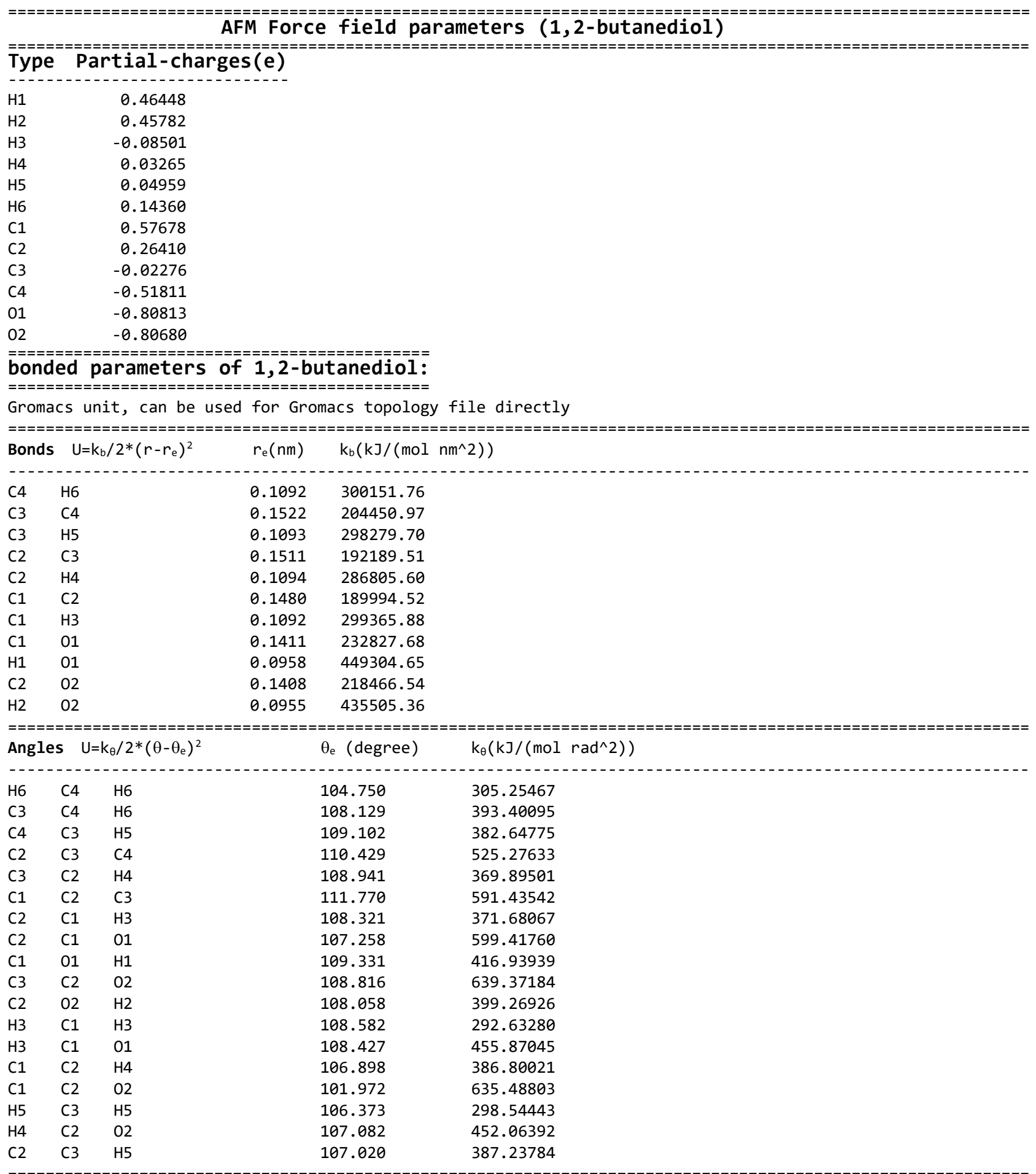

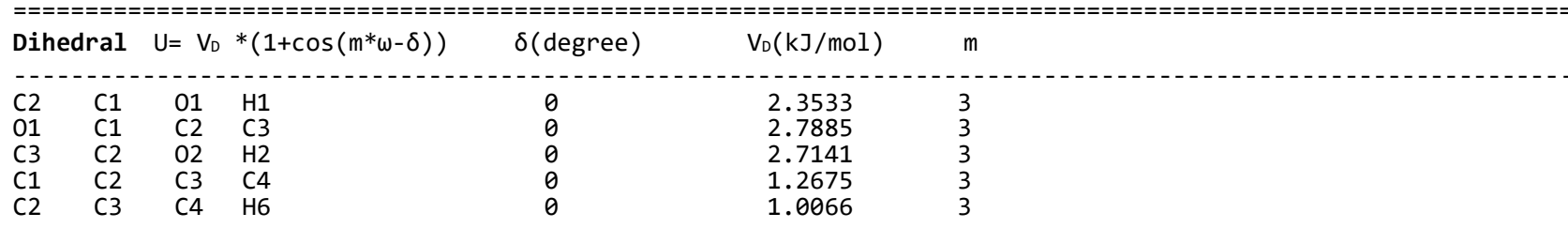

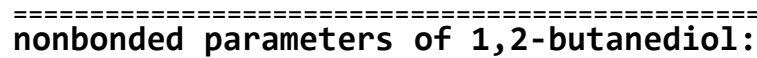

Energy expression:

$\operatorname{EXP}=A^{*} \exp \left(-\alpha^{*} r\right)$

$\mathrm{SRD}=\mathrm{C} 6 /\left(\mathrm{r}^{6}+\mathrm{R}^{6}\right)$ 


\begin{tabular}{|c|c|c|c|c|c|c|c|}
\hline atom1 & atom2 & Repulsion & $\mathrm{A}(\mathrm{kcal} / \mathrm{mol})$ & $\alpha\left(\AA^{-1}\right)$ & Dispersion & $\mathrm{C} 6\left(\mathrm{kcal} /\left(\operatorname{mol} \AA^{6}\right)\right)$ & $\mathrm{R} \theta(\AA)$ \\
\hline H1 & H3 & EXP & 5813.606 & 3.600 & & & \\
\hline H1 & C3 & EXP & 13886.622 & 3.600 & & & \\
\hline $\mathrm{H} 1$ & C4 & EXP & 30299.559 & 3.600 & & & \\
\hline $\mathrm{H} 1$ & 02 & EXP & 4871.067 & 3.600 & & & \\
\hline H2 & H3 & EXP & 688.300 & 3.600 & & & \\
\hline $\mathrm{H} 2$ & C3 & EXP & 13501.061 & 3.600 & & & \\
\hline $\mathrm{H} 2$ & C4 & EXP & 12443.744 & 3.600 & & & \\
\hline $\mathrm{H} 2$ & 01 & EXP & 2102.559 & 3.600 & & & \\
\hline H3 & $\mathrm{H} 4$ & EXP & 2358.846 & 3.600 & & & \\
\hline H3 & H5 & EXP & 2637.283 & 3.600 & & & \\
\hline H3 & H6 & EXP & 1335.943 & 3.600 & & & \\
\hline H4 & C4 & EXP & 7715.625 & 3.600 & & & \\
\hline $\mathrm{H} 4$ & 01 & EXP & 4785.966 & 3.600 & & & \\
\hline H5 & 01 & EXP & 4520.413 & 3.600 & & & \\
\hline H5 & 02 & EXP & 5033.266 & 3.600 & & & \\
\hline H6 & 01 & EXP & 3827.058 & 3.600 & & & \\
\hline H6 & 02 & EXP & 5781.423 & 3.600 & & & \\
\hline C1 & C4 & EXP & 93909.990 & 3.600 & SRD & -1767.669 & 2.045 \\
\hline C3 & 01 & EXP & 21055.548 & 3.600 & SRD & -797.150 & 1.997 \\
\hline C4 & 01 & EXP & 23885.620 & 3.600 & SRD & -711.340 & 1.997 \\
\hline C4 & 02 & EXP & 7754.335 & 3.600 & SRD & -718.362 & 1.997 \\
\hline 01 & 02 & & & & SRD & -323.379 & 1.949 \\
\hline HW & C4 & EXP & 2957.854 & 3.254 & & & \\
\hline HW & C3 & EXP & 25348.770 & 3.986 & & & \\
\hline HW & 02 & EXP & 1487.274 & 3.643 & & & \\
\hline HW & 01 & EXP & 1143.666 & 3.486 & & & \\
\hline OW & H6 & EXP & 4659.942 & 3.488 & & & \\
\hline OW & H5 & EXP & 4641.224 & 3.544 & & & \\
\hline OW & $\mathrm{H} 4$ & EXP & 4966.711 & 3.533 & & & \\
\hline HW & H3 & EXP & 1618.095 & 3.685 & & & \\
\hline OW & $\mathrm{H} 2$ & EXP & 2766.586 & 4.235 & & & \\
\hline OW & $\mathrm{H} 1$ & EXP & 410.405 & 2.751 & & & \\
\hline OW & 02 & EXP & 177289.140 & 4.039 & SRD & -426.795 & 1.949 \\
\hline OW & 01 & EXP & 487451.990 & 4.483 & SRD & -487.010 & 1.949 \\
\hline OW & C4 & EXP & 363382.280 & 4.146 & SRD & -1329.344 & 1.997 \\
\hline OW & C3 & EXP & 1283751.800 & 4.585 & SRD & -961.026 & 1.997 \\
\hline OW & C2 & EXP & 18125.175 & 3.046 & SRD & -1148.400 & 1.997 \\
\hline OW & C1 & EXP & 217033.710 & 3.816 & SRD & -1147.954 & 1.997 \\
\hline
\end{tabular}


Table S5. All force field parameters for 1,4-butanediol

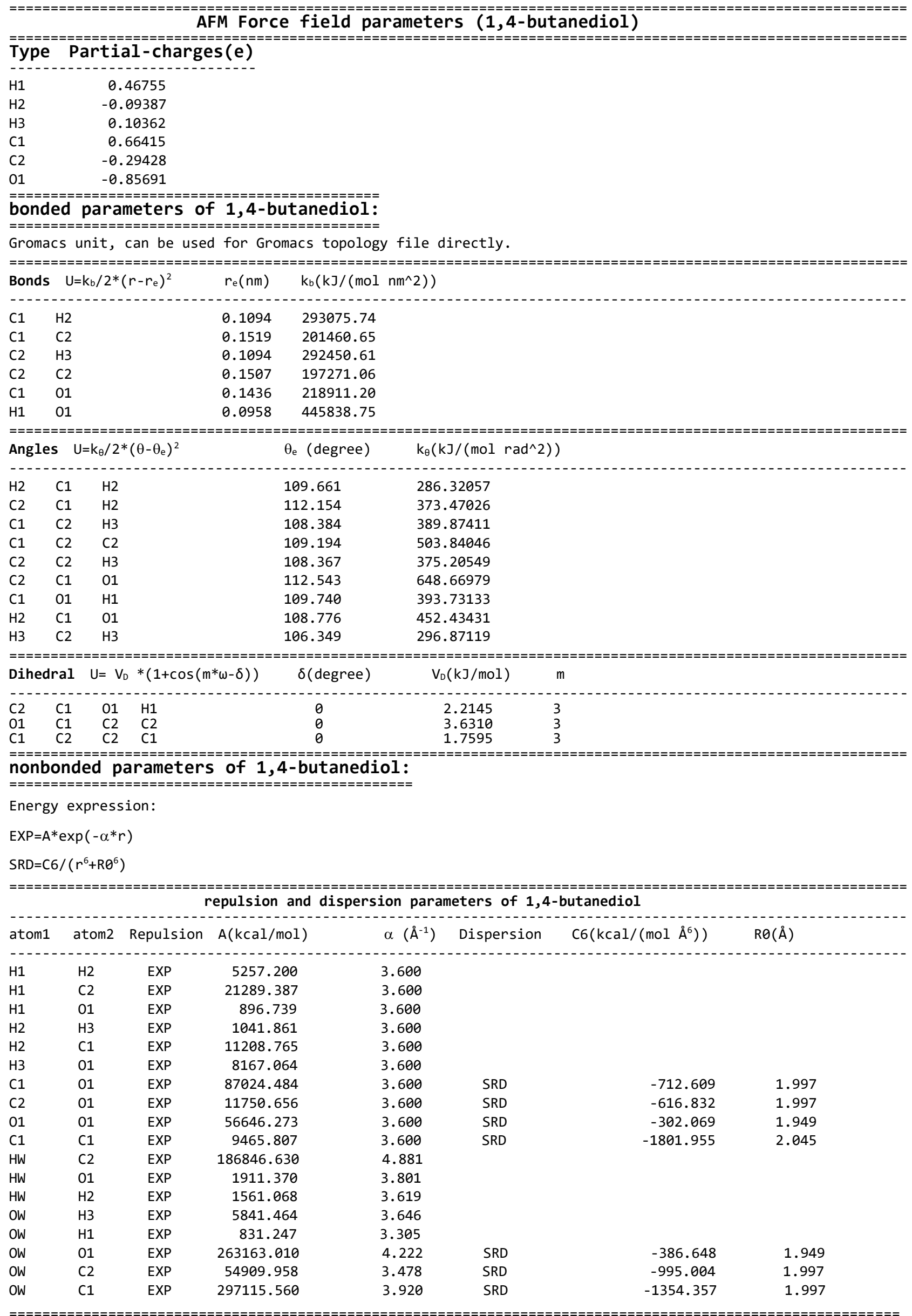


Table S6. All force field parameters for 1-hexanol

\section{AFM Force field parameters (1-hexanol)}

\begin{tabular}{|c|c|}
\hline $\mathrm{H} 1$ & 0.4410 \\
\hline $\mathrm{H} 2$ & -0.1059 \\
\hline H3 & 0.0853 \\
\hline H4 & 0.0738 \\
\hline H5 & 0.1278 \\
\hline C1 & 0.6567 \\
\hline C2 & -0.2959 \\
\hline C3 & -0.1028 \\
\hline C4 & -0.4537 \\
\hline 01 & $-0.825]$ \\
\hline
\end{tabular}

\section{$==================================$
bonded parameters of 1 -hexanol:}

$===================================$

Gromacs unit, can be used for Gromacs topology file directly.

\begin{tabular}{|c|c|c|c|}
\hline Bonds & $\mathrm{U}=\mathrm{k}_{\mathrm{b}} / 2 *\left(\mathrm{r}-\mathrm{r}_{\mathrm{e}}\right)^{2}$ & $r_{e}(n m)$ & $\mathrm{k}_{\mathrm{b}}\left(\mathrm{kJ} /\left(\mathrm{mol} \mathrm{nm}^{\wedge} 2\right)\right)$ \\
\hline C4 & H5 & 0.1093 & 294718.82 \\
\hline C3 & $\mathrm{C} 4$ & 0.1532 & 202008.54 \\
\hline C3 & C3 & 0.1532 & 195035.30 \\
\hline C3 & $\mathrm{H} 4$ & 0.1094 & 291103.53 \\
\hline C2 & C3 & 0.1530 & 196126.75 \\
\hline C2 & H3 & 0.1095 & 296298.33 \\
\hline C1 & $\mathrm{C} 2$ & 0.1523 & 200598.47 \\
\hline C1 & $\mathrm{H} 2$ & 0.1094 & 291566.63 \\
\hline C1 & 01 & 0.1435 & 208908.50 \\
\hline $\mathrm{H} 1$ & 01 & 0.0959 & 453996.95 \\
\hline
\end{tabular}

$===$

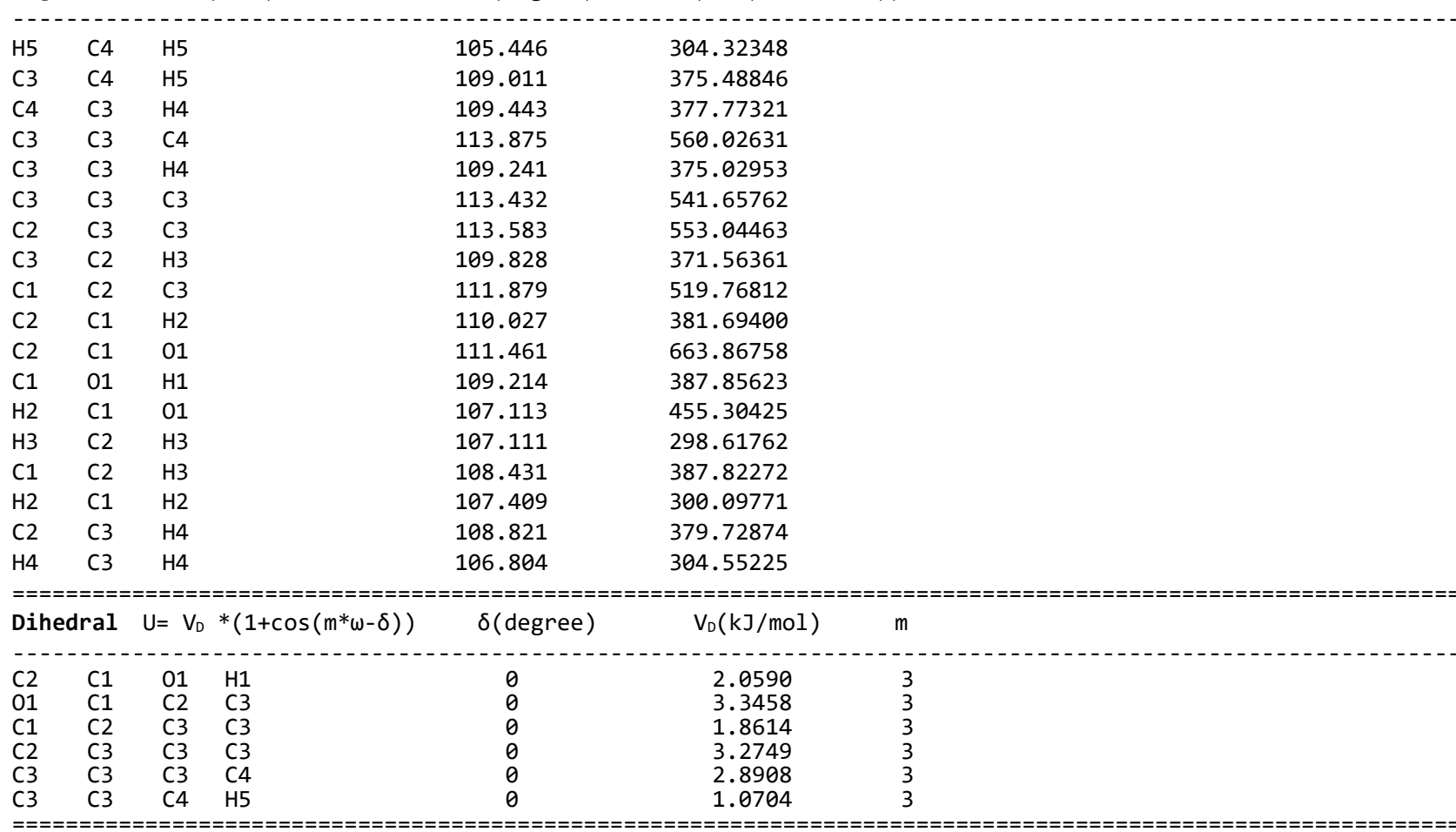

nonbonded parameters of 1-hexanol:

Energy expression:

$\operatorname{EXP}=A^{*} \exp \left(-\alpha^{*} r\right)$

$\mathrm{SRD}=\mathrm{C} 6 /\left(\mathrm{r}^{6}+\mathrm{R} \theta^{6}\right)$

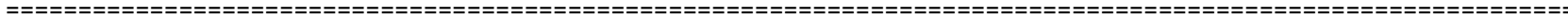
repulsion and dispersion parameters of 1-hexanol

atom1 atom2 Repulsion $\mathrm{A}(\mathrm{kcal} / \mathrm{mol}) \quad \alpha\left(\AA^{-1}\right)$ Dispersion $\mathrm{C} 6\left(\mathrm{kcal} /\left(\mathrm{mol} \AA^{6}\right)\right) \quad \mathrm{R} \theta(\AA)$ 


\begin{tabular}{|c|c|c|c|c|c|c|c|}
\hline $\mathrm{H} 1$ & $\mathrm{H} 2$ & EXP & 5380.436 & 3.600 & & & \\
\hline $\mathrm{H} 1$ & C2 & EXP & 21552.016 & 3.600 & & & \\
\hline $\mathrm{H} 1$ & C3 & EXP & 2353.680 & 3.600 & & & \\
\hline $\mathrm{H} 1$ & C4 & EXP & 11649.859 & 3.600 & & & \\
\hline $\mathrm{H} 2$ & H3 & EXP & 1940.280 & 3.600 & & & \\
\hline $\mathrm{H} 2$ & $\mathrm{H} 4$ & EXP & 1050.563 & 3.600 & & & \\
\hline $\mathrm{H} 2$ & H5 & EXP & 1152.701 & 3.600 & & & \\
\hline H3 & C3 & EXP & 5064.587 & 3.600 & & & \\
\hline H3 & C4 & EXP & 13780.302 & 3.600 & & & \\
\hline H3 & 01 & EXP & 8724.680 & 3.600 & & & \\
\hline H4 & C2 & EXP & 7085.585 & 3.600 & & & \\
\hline $\mathrm{H} 4$ & C3 & EXP & 5614.442 & 3.600 & & & \\
\hline $\mathrm{H} 4$ & C4 & EXP & 9068.208 & 3.600 & & & \\
\hline H4 & 01 & EXP & 5101.671 & 3.600 & & & \\
\hline H5 & C2 & EXP & 12890.550 & 3.600 & & & \\
\hline H5 & C3 & EXP & 4269.346 & 3.600 & & & \\
\hline H5 & 01 & EXP & 2386.313 & 3.600 & & & \\
\hline C1 & C3 & EXP & 72572.297 & 3.600 & SRD & -1349.881 & 2.045 \\
\hline C1 & C4 & EXP & 120447.900 & 3.600 & SRD & -1912.528 & 2.045 \\
\hline C2 & C3 & EXP & 63544.934 & 3.600 & SRD & -1361.720 & 2.045 \\
\hline C2 & C4 & EXP & 22582.174 & 3.600 & SRD & -1868.085 & 2.045 \\
\hline C3 & C4 & EXP & 65674.015 & 3.600 & SRD & -1642.670 & 2.045 \\
\hline C3 & 01 & EXP & 32360.299 & 3.600 & SRD & -621.845 & 1.997 \\
\hline C4 & 01 & EXP & 994.065 & 3.600 & SRD & -660.873 & 1.997 \\
\hline HW & C4 & EXP & 581.110 & 2.634 & & & \\
\hline HW & C3 & EXP & 10277.565 & 3.914 & & & \\
\hline HW & C2 & EXP & 7279.235 & 3.653 & & & \\
\hline HW & 01 & EXP & 1586.041 & 3.706 & & & \\
\hline OW & H5 & EXP & 3746.813 & 3.370 & & & \\
\hline OW & $\mathrm{H} 4$ & EXP & 4345.137 & 3.479 & & & \\
\hline OW & $\mathrm{H} 3$ & EXP & 3845.395 & 3.431 & & & \\
\hline HW & $\mathrm{H} 2$ & EXP & 1002.162 & 3.358 & & & \\
\hline OW & $\mathrm{H} 1$ & EXP & 872.215 & 3.425 & & & \\
\hline OW & 01 & EXP & 226554.690 & 4.149 & SRD & -449.113 & 1.949 \\
\hline OW & C4 & EXP & 1068268.000 & 4.519 & SRD & -1373.652 & 1.997 \\
\hline OW & C3 & EXP & 312620.840 & 4.063 & SRD & -1067.525 & 1.997 \\
\hline OW & C2 & EXP & 37899.992 & 3.391 & SRD & -1140.630 & 1.997 \\
\hline OW & C1 & EXP & 432776.020 & 4.087 & SRD & -1148.043 & 1.997 \\
\hline
\end{tabular}


Table S7. All force field parameters for Menthol

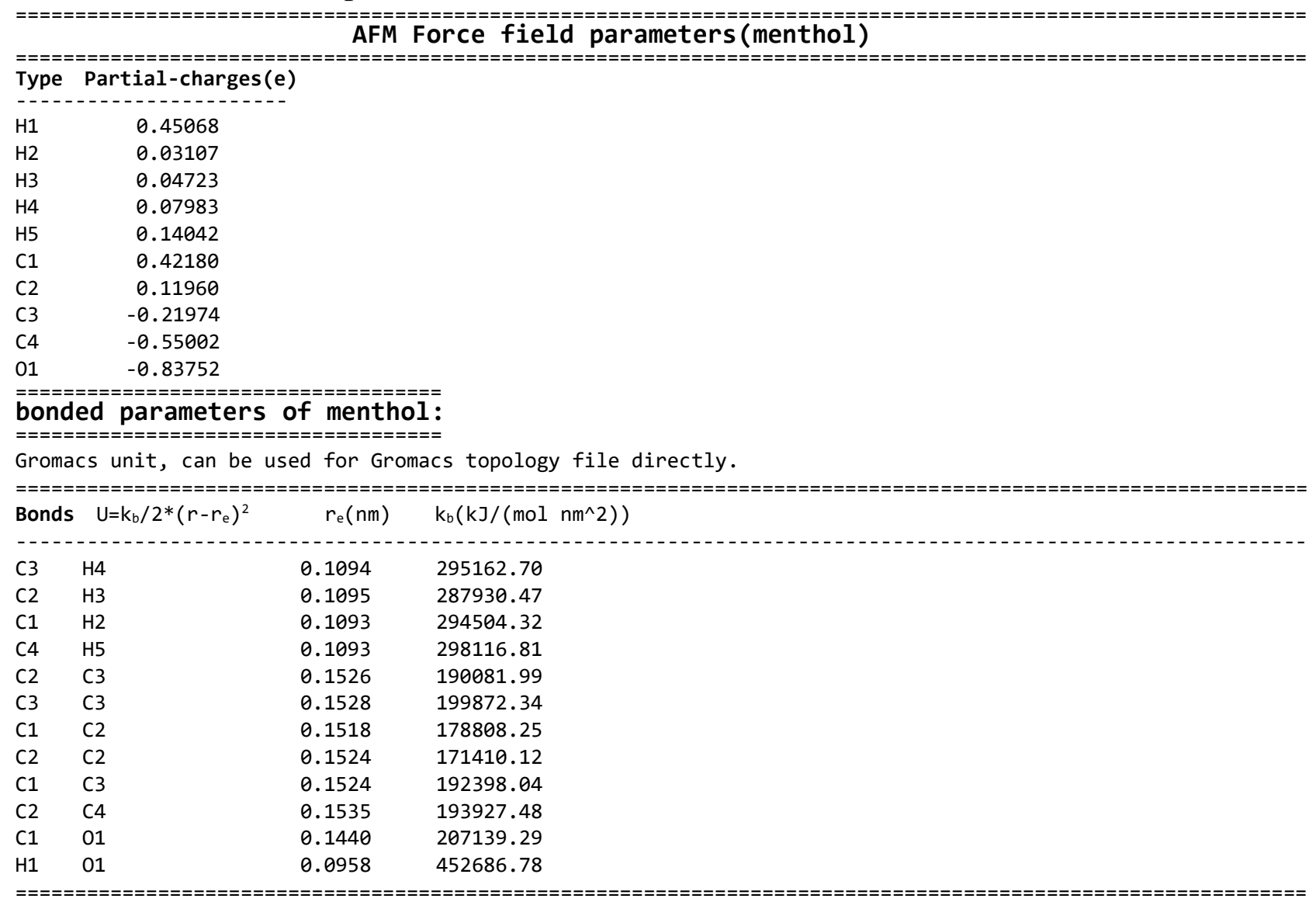

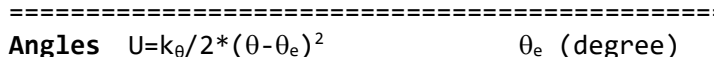

\begin{tabular}{|c|c|c|c|c|}
\hline $\mathrm{H} 4$ & $\mathrm{C} 3$ & $\mathrm{H} 4$ & 104.850 & 305.07222 \\
\hline H5 & $\mathrm{C} 4$ & H5 & 104.973 & 297.85812 \\
\hline $\mathrm{C} 2$ & C3 & $\mathrm{H} 4$ & 107.249 & 377.17131 \\
\hline $\mathrm{C} 3$ & $\mathrm{C} 3$ & $\mathrm{H} 4$ & 107.792 & 383.74597 \\
\hline $\mathrm{C} 3$ & $\mathrm{C} 2$ & H3 & 108.917 & 378.62868 \\
\hline $\mathrm{C} 1$ & $\mathrm{C} 2$ & H3 & 106.733 & 380.69330 \\
\hline $\mathrm{C} 2$ & $\mathrm{C} 2$ & $\mathrm{H} 3$ & 107.556 & 369.41581 \\
\hline $\mathrm{C} 2$ & $\mathrm{C} 1$ & $\mathrm{H} 2$ & 110.661 & 351.01967 \\
\hline $\mathrm{C} 3$ & $\mathrm{C} 1$ & $\mathrm{H} 2$ & 110.114 & 375.02770 \\
\hline $\mathrm{C} 1$ & C3 & $\mathrm{H} 4$ & 106.123 & 386.13317 \\
\hline $\mathrm{C} 4$ & $\mathrm{C} 2$ & H3 & 108.822 & 379.23695 \\
\hline $\mathrm{C} 2$ & C4 & H5 & 108.156 & 380.17340 \\
\hline $\mathrm{C} 2$ & $\mathrm{C} 3$ & $\mathrm{C} 3$ & 111.601 & 553.97288 \\
\hline $\mathrm{C} 1$ & $\mathrm{C} 2$ & C3 & 111.436 & 531.05886 \\
\hline $\mathrm{C} 2$ & $\mathrm{C} 2$ & $\mathrm{C} 3$ & 111.201 & 556.26352 \\
\hline $\mathrm{C} 1$ & $\mathrm{C} 2$ & $\mathrm{C} 2$ & 110.595 & 561.60836 \\
\hline $\mathrm{C} 2$ & $\mathrm{C} 1$ & C3 & 114.357 & 519.12640 \\
\hline $\mathrm{C} 1$ & C3 & $\mathrm{C} 2$ & 110.768 & 570.03421 \\
\hline $\mathrm{C} 3$ & $\mathrm{C} 2$ & $\mathrm{C} 3$ & 110.649 & 550.98424 \\
\hline $\mathrm{C} 3$ & $\mathrm{C} 2$ & $\mathrm{C} 4$ & 112.740 & 562.79285 \\
\hline $\mathrm{C} 2$ & $\mathrm{C} 2$ & $\mathrm{C} 4$ & 110.171 & 531.99943 \\
\hline $\mathrm{C} 4$ & $\mathrm{C} 2$ & $\mathrm{C} 4$ & 113.644 & 607.00286 \\
\hline $\mathrm{C} 2$ & $\mathrm{C} 1$ & 01 & 108.075 & 597.21003 \\
\hline $\mathrm{C} 3$ & $\mathrm{C} 1$ & 01 & 110.579 & 670.19445 \\
\hline $\mathrm{H} 2$ & $\mathrm{C} 1$ & 01 & 106.906 & 452.09397 \\
\hline C1 & 01 & $\mathrm{H} 1$ & 109.656 & 384.24673 \\
\hline
\end{tabular}

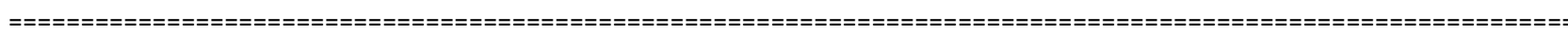

Dihedral $\mathrm{U}=\mathrm{V}_{\mathrm{D}} *(1+\cos (\mathrm{m} * \omega-\delta)) \quad \delta($ degree $) \quad \mathrm{V}_{\mathrm{D}}(\mathrm{kJ} / \mathrm{mol}) \quad \mathrm{m}$

\begin{tabular}{|c|c|c|c|c|c|c|}
\hline $\begin{array}{l}\text { C3 } \\
\text { C2 } \\
\text { C2 } \\
\text { C2 } \\
\text { C3 } \\
\text { C1 } \\
\text { C4 } \\
\text { C4 }\end{array}$ & $\begin{array}{l}\text { C2 } \\
\text { C2 } \\
\text { C2 } \\
\text { C3 } \\
\text { C1 } \\
\text { C2 } \\
\text { C2 } \\
\text { C2 }\end{array}$ & $\begin{array}{l}\text { C4 } \\
\text { C4 } \\
\text { C3 } \\
\text { C3 } \\
\text { C2 } \\
\text { C2 } \\
\text { C3 } \\
\text { C3 }\end{array}$ & $\begin{array}{l}\mathrm{H} 5 \\
\mathrm{H} 5 \\
\mathrm{C} 3 \\
\mathrm{C} 2 \\
\mathrm{C} 2 \\
\mathrm{C} 4 \\
\mathrm{C} 1 \\
\mathrm{C} 3\end{array}$ & $\begin{array}{l}0 \\
0 \\
0 \\
0 \\
0 \\
0 \\
0 \\
0\end{array}$ & $\begin{array}{r}1.5700 \\
1.1149 \\
3.7281 \\
3.2342 \\
-0.2097 \\
0.8152 \\
3.9132 \\
1.9666\end{array}$ & $\begin{array}{l}3 \\
3 \\
3 \\
3 \\
3 \\
3 \\
3 \\
3\end{array}$ \\
\hline
\end{tabular}




\begin{tabular}{|c|c|c|c|c|c|c|c|}
\hline \multicolumn{8}{|c|}{ 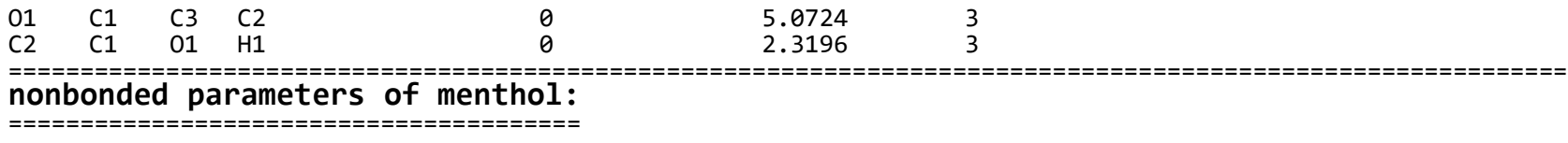 } \\
\hline \multicolumn{8}{|c|}{ Energy expression: } \\
\hline \multicolumn{8}{|c|}{$E X P=A^{*} \exp \left(-\alpha^{*} r\right)$} \\
\hline \multicolumn{8}{|c|}{$S R D=C 6 /\left(r^{6}+R \theta^{6}\right)$} \\
\hline \multicolumn{8}{|c|}{ 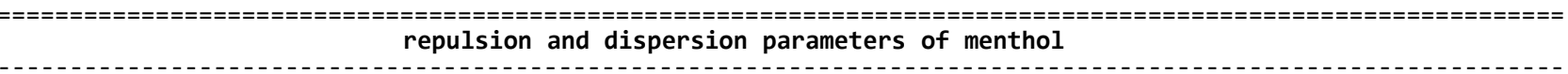 } \\
\hline atom1 & atom2 & Repulsion & $\mathrm{A}(\mathrm{kcal} / \mathrm{mol})$ & $\alpha\left(\AA^{-1}\right)$ & Dispersion & $\mathrm{C} 6\left(\mathrm{kcal} /\left(\operatorname{mol} \AA^{6}\right)\right)$ & $\mathrm{R} 0(\AA)$ \\
\hline OW & H5 & EXP & 4975.458 & 3.528 & & & \\
\hline $\mathrm{OW}$ & $\mathrm{H} 4$ & EXP & 4565.544 & 3.465 & & & \\
\hline OW & H3 & EXP & 4621.373 & 3.516 & & & \\
\hline OW & $\mathrm{H} 2$ & EXP & 115550.940 & 5.184 & & & \\
\hline $\mathrm{OW}$ & $\mathrm{H} 1$ & EXP & 1030.417 & 3.677 & & & \\
\hline $\mathrm{HW}$ & 01 & EXP & 2026.660 & 3.896 & & & \\
\hline $\mathrm{OW}$ & 01 & EXP & 127779.530 & 3.881 & SRD & -490.062 & 1.950 \\
\hline $\mathrm{HW}$ & C4 & EXP & 1840.926 & 3.000 & & & \\
\hline OW & $\mathrm{C} 4$ & EXP & 130643.640 & 3.802 & SRD & -1280.616 & 1.997 \\
\hline $\mathrm{HW}$ & $\mathrm{C} 3$ & EXP & 4221.927 & 3.305 & & & \\
\hline OW & C3 & EXP & 1878096.500 & 4.776 & SRD & -1062.714 & 1.997 \\
\hline OW & $\mathrm{C} 2$ & EXP & 157344.300 & 3.877 & SRD & -894.089 & 1.997 \\
\hline OW & $\mathrm{C} 1$ & EXP & 17066.723 & 3.000 & SRD & -911.586 & 1.997 \\
\hline H1 & $\mathrm{C} 3$ & EXP & 12367.432 & 3.600 & & & \\
\hline $\mathrm{H} 1$ & $\mathrm{C} 4$ & EXP & 14327.950 & 3.600 & & & \\
\hline $\mathrm{H} 2$ & C4 & EXP & 7760.899 & 3.600 & & & \\
\hline H3 & 01 & EXP & 5886.729 & 3.600 & & & \\
\hline H3 & $\mathrm{C} 3$ & EXP & 6015.088 & 3.600 & & & \\
\hline H3 & C4 & EXP & 11212.040 & 3.600 & & & \\
\hline $\mathrm{H} 4$ & 01 & EXP & 8529.961 & 3.600 & & & \\
\hline $\mathrm{H} 4$ & $\mathrm{C} 3$ & EXP & 10212.848 & 3.600 & & & \\
\hline H4 & C4 & EXP & 10277.057 & 3.600 & & & \\
\hline H5 & 01 & EXP & 5700.941 & 3.600 & & & \\
\hline H5 & $\mathrm{C} 3$ & EXP & 10113.108 & 3.600 & & & \\
\hline H5 & C4 & EXP & 11731.186 & 3.600 & & & \\
\hline $\mathrm{C} 1$ & $\mathrm{C} 3$ & EXP & 109671.750 & 3.600 & SRD & -1240.321 & 2.045 \\
\hline C1 & C4 & EXP & 114943.500 & 3.600 & SRD & -1524.774 & 2.045 \\
\hline $\mathrm{C} 2$ & $\mathrm{C} 3$ & EXP & 60425.850 & 3.600 & SRD & -851.391 & 2.045 \\
\hline C2 & $\mathrm{C} 4$ & EXP & 23047.958 & 3.600 & SRD & -1088.027 & 2.045 \\
\hline 01 & $\mathrm{C} 2$ & EXP & 28392.861 & 3.600 & SRD & -586.663 & 1.997 \\
\hline 01 & $\mathrm{C} 3$ & & & & SRD & -751.036 & 1.997 \\
\hline 01 & $\mathrm{C} 4$ & EXP & 5641.582 & 3.600 & SRD & -838.632 & 1.997 \\
\hline$C 2$ & $\mathrm{C} 2$ & EXP & 43914.349 & 3.600 & & & \\
\hline C3 & $\mathrm{C} 3$ & EXP & 40040.042 & 3.600 & SRD & -1229.016 & 2.045 \\
\hline C3 & $\mathrm{C} 4$ & EXP & 58938.774 & 3.600 & SRD & -1815.965 & 2.045 \\
\hline C4 & C4 & EXP & 27.441 & 3.600 & SRD & -2302.476 & 2.045 \\
\hline
\end{tabular}


Table S8. All force field parameters for piperidine

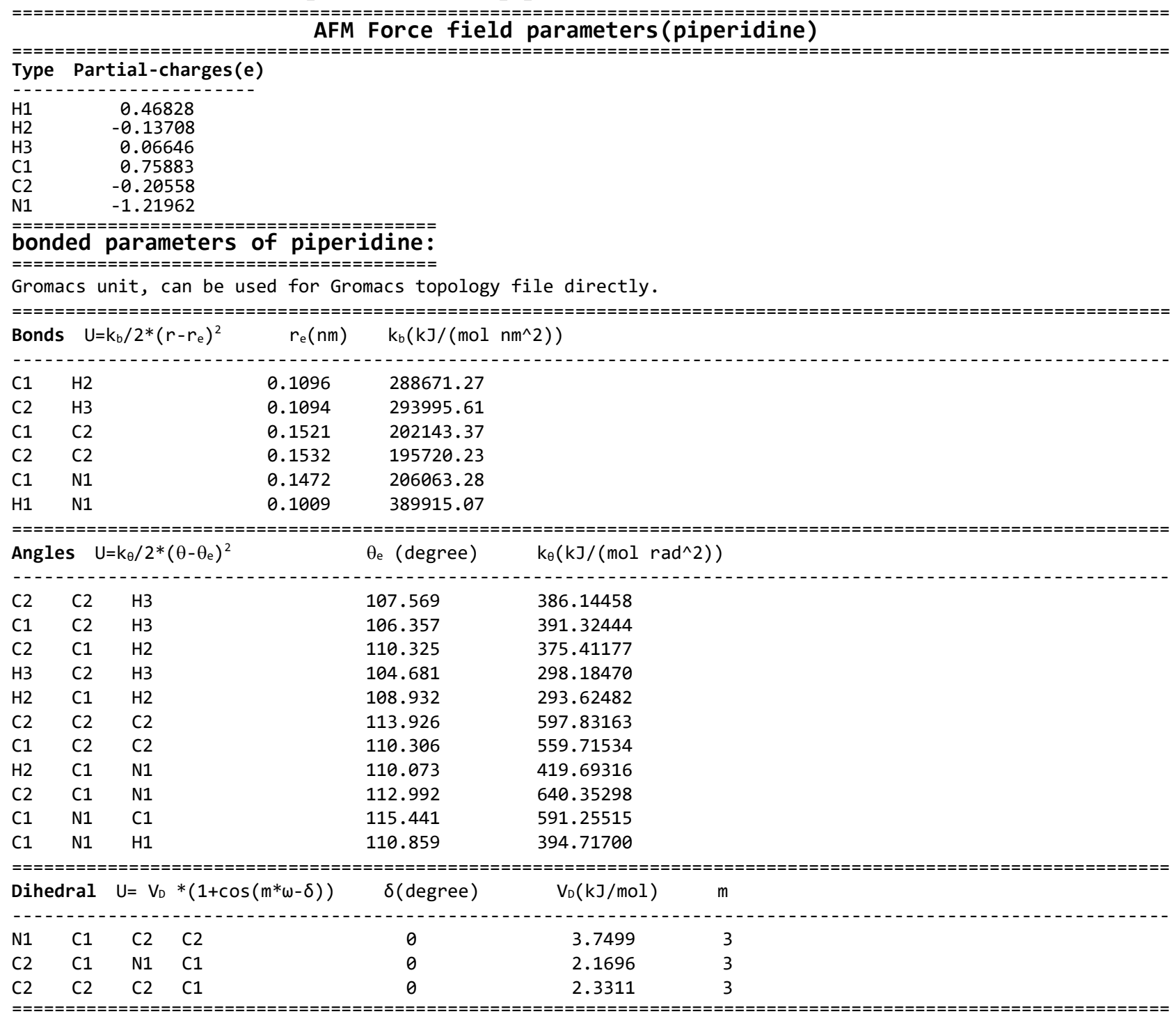

\section{nonbonded parameters of piperidine:}

$===============$
Energy expression:

$E X P=A^{*} \exp \left(-\alpha^{*} r\right)$

$\mathrm{SRD}=\mathrm{C} 6 /\left(r^{6}+\mathrm{R}^{6}\right)$

\begin{tabular}{|c|c|c|c|c|c|c|c|}
\hline atom1 & atom2 & Repulsion & $\mathrm{A}(\mathrm{kcal} / \mathrm{mol})$ & $\alpha\left(\AA^{-1}\right)$ & Dispersion & $\mathrm{C} 6\left(\mathrm{kcal} /\left(\operatorname{mol} \AA^{6}\right)\right)$ & $\mathrm{R} \theta(\AA)$ \\
\hline HW & $\mathrm{C} 2$ & EXP & 12504.032 & 3.688 & & & \\
\hline $\mathrm{HW}$ & N1 & EXP & 377.804 & 2.780 & & & \\
\hline OW & H3 & EXP & 3338.797 & 3.378 & & & \\
\hline HW & $\mathrm{H} 2$ & EXP & 894.973 & 3.314 & & & \\
\hline OW & $\mathrm{H} 1$ & EXP & 973.422 & 2.996 & & & \\
\hline OW & N1 & EXP & 753771.250 & 4.429 & SRD & -586.507 & 1.984 \\
\hline OW & $\mathrm{C} 2$ & EXP & 227087.430 & 4.024 & SRD & -1089.250 & 1.997 \\
\hline OW & $\mathrm{C} 1$ & EXP & 300912.670 & 3.927 & SRD & -1207.173 & 1.997 \\
\hline N1 & $\mathrm{C} 2$ & EXP & 18157.196 & 3.600 & SRD & -631.253 & 2.032 \\
\hline N1 & H3 & EXP & 8145.256 & 3.600 & & & \\
\hline $\mathrm{C} 1$ & $\mathrm{C} 2$ & EXP & 76466.024 & 3.600 & SRD & -1482.182 & 2.045 \\
\hline $\mathrm{C} 1$ & $\mathrm{H} 2$ & EXP & 22623.700 & 3.600 & & & \\
\hline $\mathrm{C} 2$ & $\mathrm{H} 1$ & EXP & 29692.466 & 3.600 & & & \\
\hline $\mathrm{C} 2$ & H3 & EXP & 3404.436 & 3.600 & & & \\
\hline $\mathrm{H} 1$ & $\mathrm{H} 2$ & EXP & 6641.957 & 3.600 & & & \\
\hline $\mathrm{H} 2$ & H3 & EXP & 2553.736 & 3.600 & & & \\
\hline
\end{tabular}




\section{The experimental enthalpy of hydration of 1,2-butanediol based on Suleiman}

and Eckert data

The activity coefficients of 1,2-butanediol at infinite dilution, $\gamma_{s}^{\infty}$, in water were taken from the work of Suleiman and Eckert. ${ }^{1}$ (Note that the Suleiman and Eckert work only report the $\gamma_{s}^{\infty}$ with two significant numbers. The values at $338.6 \mathrm{~K}$ and $348.4 \mathrm{~K}$ are identical within two significant numbers.

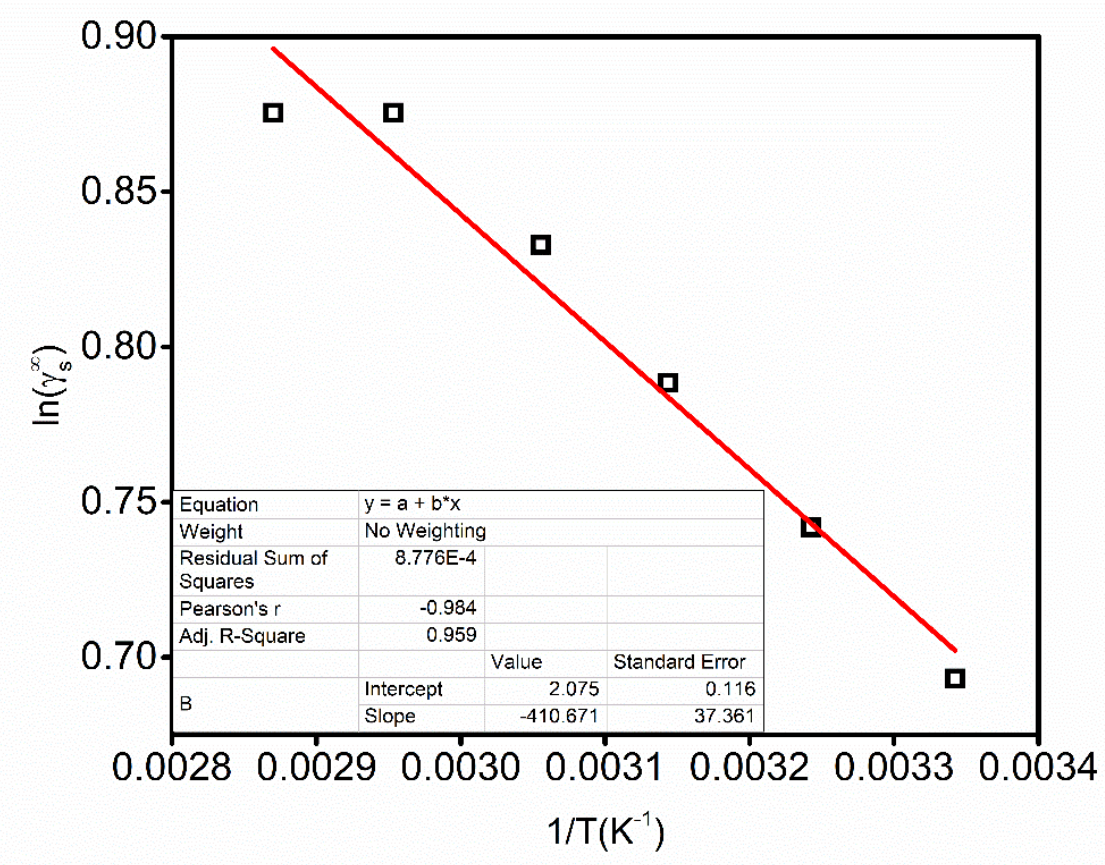

Figure S1. The plot of $\ln \gamma_{s}^{\infty} \sim 1 / \mathrm{T}$. A linear regression gives a slope of $-410.67 \mathrm{~K}$, which is $\frac{\Delta H_{s o l}}{R}$ according to the van't Hoff Equation. This gives a $\Delta H_{\text {sol }}$ of $-3.4 \mathrm{~kJ} / \mathrm{mol}$. 


\section{Reference}

1. Suleiman, D.; Eckert, C. A., Limiting Activity Coefficients of Diols in Water by a Dew Point Technique. J. Chem. Eng. Data 1994, 39, 692-696. 